PROCEEDINGS OF THE

AMERICAN MATHEMATICAL SOCIETY

Volume 135, Number 11, November 2007, Pages 3745-3753

S 0002-9939(07)08954-X

Article electronically published on June 21, 2007

\title{
CIRCLE-SUM AND MINIMAL GENUS SURFACES IN RULED 4-MANIFOLDS
}

\author{
BANG-HE LI AND TIAN-JUN LI
}

(Communicated by Daniel Ruberman)

\begin{abstract}
We describe a circle-sum construction of smoothly embedded surface in a smooth 4-manifold. We apply this construction to give a simpler solution of the minimal genus problem for nontrivial $S^{2}$ bundles over surfaces. We also treat the case of blow-ups.
\end{abstract}

\section{INTRODUCTION}

Given two connected embedded surfaces in a smooth 4-manifold, we can construct a new connected embedded surface by simply tubing them. However, if there is a nicely embedded cylinder connecting two nonseparating circles, then we can get a new surface with lower genus. In this note we explicitly describe such a construction, which we call the circle-sum construction. This construction should be compared with the famous Whitney trick. Recall that the Whitney trick can be applied to cancel a pair of intersection points with opposite sign in the presence of a nicely embedded disk.

In [4, the minimal genus problem for $S^{2}$ bundles over surfaces of positive genus was completely solved. However, for nontrivial bundles, the Whitney trick part of the proof (case II for Theorem 2) was rather complicated. We will give a much simpler proof by instead using the circle-sum construction. Moreover, the circlesum construction can be similarly applied to blow-ups.

We thank H. Gao and X. Zhao for their interest in this work. We are very grateful to the referee for pointing out a mistake and suggesting how to fix it.

\section{The CIRCLE-SUM CONSTRUCTION}

Let $S_{0}$ and $S_{1}$ be two disjoint smooth connected oriented closed embedded surfaces of positive genus $g_{0}$ and $g_{1}$ in a smooth 4-manifold $M$ representing the homology classes $\xi_{0}$ and $\xi_{1}$, respectively. Let $g_{i}: S^{1} \rightarrow S_{i}, i=0,1$, be a smooth oriented circle representing a nonzero class in $H_{1}\left(S_{i} ; \mathbb{Z}\right)$. Write $S_{i}^{1}=g_{i}\left(S^{1}\right)$. Let $e_{i}, i=0,1$, be a normal section of $S_{i}^{1} \subset S_{i}$ such that the orientation of $S_{0}$ along $S_{0}^{1}$ is given by the orientation of $S_{0}^{1}$ together with $e_{0}$, while the orientation of $S_{1}$ along $S_{1}^{1}$ is given by the orientation of $S_{1}^{1}$ together with $-e_{1}$.

Received by the editors August 3, 2006.

2000 Mathematics Subject Classification. Primary 57R40, 57R57; Secondary 57R17.

The first author was supported in part by 973 project (2004CB318000).

The second author was supported in part by NSF and the McKnight Foundation.

(C)2007 American Mathematical Society Reverts to public domain 28 years from publication 3745 
Theorem 2.1. Suppose that there exists a smooth embedding of a closed annulus

$$
f: S^{1} \times[0,1] \longrightarrow M
$$

such that for $i=0,1$,

$$
\begin{array}{ll}
\left.f\right|_{S^{1} \times\{i\}} & =g_{i}, \\
f\left(S^{1} \times(0,1)\right) \cap S_{i} & =\emptyset, \\
T_{f(x, i)} f\left(S^{1} \times[0,1]\right) \cap T_{f(x, i)} S_{i} & =T_{f(x, i)} S_{i}^{1},
\end{array}
$$

- there exists a nonzero section e of the normal bundle of the annulus $f\left(S^{1} \times[0,1]\right) \subset M$ with

$$
\left.e\right|_{S_{i}^{1}}=e_{i} \text { or }-e_{i}
$$

Then there exists a smooth connected oriented closed embedded surface of genus $g_{0}+g_{1}-1$ representing the class $\xi_{0}+\xi_{1}$.

Proof. The idea is as follows. We first remove open annular neighborhoods of the circle $S_{i}^{1}$ in each surface leaving two circles as a boundary in each surface. We then use two pushoffs via the framing $e$ of the embedded annulus to glue in two annuli at the boundary. This gives a surface $S$ with the same Euler characteristic as for the original disjoint union $S_{0} \amalg S_{1}$. Since $S_{i}^{1}, i=0,1$, represents a nonzero class in $H_{1}\left(S_{i} ; \mathbb{Z}\right)$, the surface $S$ is easily seen to be connected. We then conclude from the Euler characteristic computation

$$
2 g(S)-2=\left(2 g\left(S_{0}\right)-2\right)+\left(2 g\left(S_{1}\right)-2\right.
$$

that

$$
g(S)=g\left(S_{0}\right)+g\left(S_{1}\right)-1 .
$$

More precisely, we construct from the normal section $e$ on the annulus a smooth embedding

$$
\tilde{f}: S^{1} \times[0,1] \times[-1,1] \longrightarrow M
$$

such that for $i=0,1$,

$$
\begin{array}{lll}
\left.\tilde{f}\right|_{S^{1} \times\{i\} \times\{0\}} & = & g_{i}, \\
\tilde{f}\left(S^{1} \times\{i\} \times[-1,1]\right) & \subset & S_{i}, \\
\tilde{f}\left(S^{1} \times(0,1) \times[-1,1]\right) \cap S_{i} & = & \emptyset .
\end{array}
$$

Then

$$
S=\left(S_{0} \cup S_{1} \cup \tilde{f}\left(S^{1} \times[0,1] \times[-1,1]\right)\right)-\tilde{f}\left(S^{1} \times[0,1] \times(-1,1)\right)
$$

is a connected closed surface embedded in $M$. By smoothing the corners, we may assume the embedding is smooth.

Now we discuss orientations. First of all notice that any nowhere zero vector field $v$ in an orientable plane bundle over $S^{1}$ is homotopic to $-v$ through nowhere zero vector fields. Such a homotopy can be e.g. defined using rotations if an inner product is introduced. Thus by possibly reparametrizing the interval $[0,1]$ and deforming $e$ at the two ends we can assume that

$$
\left.e\right|_{S_{0}^{1}}=e_{0},\left.\quad e\right|_{S_{1}^{1}}=-e_{1} .
$$

Orient $S$ by extending the orientation of

$$
S \cap S_{0}=S_{0}-\tilde{f}\left(S^{1} \times\{0\} \times(-1,1)\right)
$$


inherited from that of $S_{0}$. By our assumption on $e_{0}$, on the 0-end of the two annuli this orientation is given by the orientation of $S^{1}$ together with an outward pointing vector. Thus on the 1 -end of the two annuli this orientation is given by the orientation of $S^{1}$ together with an inward pointing vector. By our assumption on $e_{1}$ this orientation agrees with the one on $S \cap S^{1}$.

Therefore the class $\xi$ represented by $S$ is $\xi_{0}+\xi_{1}$. This is justified by the natural cobordism between $S_{0} \coprod S_{1}$ and $S$ provided by the embedded thickened annulus $\tilde{f}: S^{1} \times[0,1] \times[-1,1]$.

Remark 2.2. Notice that the connected sum $S_{0} \# S_{1}$ has genus $g_{0}+g_{1}$. Thus the circle-sum construction, when it can be applied, gives a better bound for the minimal genus problem.

Remark 2.3. As pointed out by the referee it is necessary to assume the existence of the framing $e$. One can choose a framing of the normal bundle at one end of the annulus to match up with that given by a normal to the circle in the first surface. This choice determines the framing up to homotopy on the other end of the annulus, and the fact $\pi_{1}(S O(2))=\mathbb{Z}$ says that it need not match up at the other end.

Remark 2.4. However in our application we are actually given a $\tilde{f}$ satisfying (2.1). Then the framing $e=\tilde{f}^{\prime}\left(\frac{\partial}{\partial t}\right)$ of the annulus $\tilde{f}\left(S^{1} \times[0,1] \times\{0\}\right)$, where $t$ is the variable of $[-1,1]$, automatically satisfies the condition of Theorem 2.1.

\section{Nontrivial $S^{2}$-BUndLes AND BLOW-UPS}

3.1. Nontrivial $S^{2}$-bundles. Let $\Sigma$ be a connected oriented surface of positive genus, and $N$ be the nontrivial $S^{2}$-bundle over $\Sigma$. Notice that such a bundle is unique as $\pi_{1}(S O(3))=\mathbb{Z}_{2}$.

Think of $N$ as the projectivization $\mathbb{P}(\eta \oplus \mathbb{C})$, where $\eta$ is a complex line bundle with Chern number 1 and orient $N$ this way. Let $f$ be a complex oriented fiber and $y$ its homology class. Let $S_{0}$ be the zero section of $\eta$ and $x$ its homology class. Clearly

$$
x \cdot x=1, \quad x \cdot y=1, \quad y \cdot y=0,
$$

and $\{x, y\}$ is a basis of $H_{2}(N ; \mathbb{Z})$.

Let $S_{\infty}$ be the infinity section and $x^{\prime}$ its homology class. Let $\phi$ be the antipodal map on the fibers. Then $\phi$ is an orientation-reversing, and $\phi$ interchanges $S_{0}$ and $S_{\infty}$. Therefore

$$
x^{\prime} \cdot x^{\prime}=\phi_{*}(x) \cdot \phi_{*}(x)=-1 .
$$

On the other hand, $x^{\prime}=x+c y$ for some integer $c$ as $x^{\prime} \cdot y=1$. Therefore $(x+c y) \cdot(x+c y)=1+2 c=-1$, which implies that

$$
y=x-x^{\prime} .
$$

Theorem 2 in [4] can be restated as

Theorem 3.1. The minimal genus of $\xi=a x+b y$ is given by

$$
g_{\xi}= \begin{cases}a^{\prime} g+\frac{\left(a^{\prime}-1\right)\left(\left|a^{\prime}+2 b^{\prime}\right|-2\right)}{2}, & \text { if } a \neq 0, \\ 0, & \text { if } a=0,\end{cases}
$$

where $a^{\prime}=|a|$ and $b^{\prime}=\frac{a}{|a|} b$ if $a \neq 0$. 
Proof. First of all, notice that $\phi_{*}(y)=-y$, and hence by (3.1),

$$
\phi_{*}(\xi)=\phi_{*}(a x+b y)=a x^{\prime}-b y=a(x-y)-b y=a x-(a+b) y .
$$

In particular, the right hand side of (3.2) is the same for $\xi$ and $\phi_{*}(\xi)$. As $\phi$ is an orientation-reversing diffeomorphism, we can thus reduce to the case that $\xi^{2} \geq 0$, i.e.

$$
(a x+b y)^{2}=a^{2}+2 a b=a(a+2 b) \geq 0 .
$$

It then follows from the adjunction inequality in [6], applied with the standard canonical class $K=P D(-2 x+(2 g-1) y)$, that $g_{\xi}$ is bounded by (3.2) for such $\xi$. Thus we only need to construct an embedded surface in the class $\xi$ with genus $g_{\xi}$.

We next make a further reduction to the case $a \geq 0$. This is possible as the automorphism -identity is realized by an orientation-preserving diffeomorphism. To construct such a diffeomorphism we start with an orientation-reversing diffeomorphism $h$ of the base $\Sigma$. The pull back of the $S^{2}$-bundle $N$ is still a nontrivial $S^{2}$-bundle, hence we get a diffeomorphism $\psi$ of $N$. Notice that $\psi$ is orientationreversing and $\psi_{*}(y)=y$. It is easy to see, as in the case of $\phi$, that $\psi_{*}(x)=-x+y$. Now the composition $\phi \circ \psi$ has the effect of -identity on $H_{2}(N ; \mathbb{Z})$ (see also [1). Since the right hand side of (3.2) is the same for $\xi$ and $-\xi$, we can assume that $a \geq 0$. Moreover, as $\xi^{2}$ is already assumed to be nonnegative, we have $a+2 b \geq 0$ by (3.3).

The construction in the case $a=0$, or $a>0$ and $b \geq 0$ is straightforward and is the same as in [3. For completeness we reproduce it here.

When $a=0$ we just connect the sum of the $|b|$ fibers to obtain a sphere. Assume now $a>0$ and $b \geq 0$. Then consider the configuration of surfaces with $b$ fibers and $a$ sections. We can certainly choose such a configuration with only positive intersection points, the total number being

$$
a b+\frac{a(a-1)}{2} .
$$

Do surgery on $a+b-1$ of those to make the configuration into an irreducible nodal surface of genus $a g$. Do surgery on the remaining ones to obtain an embedded surface of genus exactly

$$
\begin{aligned}
& a g+a b+\frac{a(a-1)}{2}-(a+b-1) \\
= & a g+\frac{(a-1)(a+2 b-2)}{2} .
\end{aligned}
$$

Now let us look at the interesting case

$$
a>0, \quad b<0, \quad a+2 b \geq 0 .
$$

According to (3.1) we can write

$$
a x+b y=(a+2 b) x+(-b)\left(x+x^{\prime}\right) .
$$

Let $D$ be a smoothly embedded disk in $\Sigma$ parametrized by $\{z \in \mathbb{C}|| z \mid \leq 1\} . \eta$ is glued by two trivial bundles over $(\Sigma-\operatorname{Interior}(D))$ and $D$ with transition function

$$
\phi\left(e^{i \theta}, z\right)=\left(e^{i \theta}, e^{i \theta} z\right) .
$$

Let $S_{j}, j=0,1, \ldots, a+2 b$, be the sections of $\eta$ given by

$$
S_{j}(s)= \begin{cases}(s, j) & \in(\Sigma-\text { Interior }(D)) \times \mathbb{C}, \\ (z, j z) & \in D \times \mathbb{C} .\end{cases}
$$


Then each $S_{j}$ represents $x$. Let $\gamma$ be a simple closed loop in $(\Sigma-\operatorname{Interior}(D))$ representing a nonzero class in $H_{1}(\Sigma ; \mathbb{Z})$.

Observe that over $(\Sigma-$ Interior $(D)), S_{\infty}$ corresponds to the set of pairs $(s, \infty)$. Let $J=\{0,1, \ldots, a+2 b, \infty\}$. It then follows from (3.4) that for any pair of surfaces $S_{j}, S_{j^{\prime}}$ with $j, j^{\prime} \in J$ and the corresponding lifts $\gamma_{j}, \gamma_{j^{\prime}}$ of the circle $\gamma$, there is an embedding $\tilde{f}$ satisfying (2.1) by choosing an arc connecting $j$ and $j^{\prime}$ in $\mathbb{C} \cup \infty$. Thus by Remark 2.4 we can circle sum $S_{j}$ and $S_{j^{\prime}}$.

In particular, by circle-summing $S_{a+2 b}$ and $S_{\infty}$ we get a surface $S$ representing $x+x^{\prime}$. By choosing this arc to be disjoint from $J-\{a+2 b, \infty\}$, we can further assume that $S$ intersects $S_{j}, 0 \leq j \leq a+2 b-1$, only at $(0,0) \in D \times \mathbb{C}$ transversely.

In the following we will frequently encounter a collection of surfaces as the $S_{j}$ above. By the same argument we could perform the circle sum construction on such surfaces.

Since $\left(x+x^{\prime}\right)^{2}=0$, the normal bundle of $S$ is trivial. So $-b\left(x+x^{\prime}\right)$ can be represented by disjoint union of surfaces

$$
S_{1}^{\prime}, S_{2}^{\prime}, \ldots, S_{-b}^{\prime}
$$

in a small tubular neighborhood of $S$, each of which is homotopic to $S$ and intersects with each $S_{j}, 0 \leq j \leq a+2 b-1$, transversely only at one point.

In the case of $a+2 b=0$, circle-summing $S_{1}^{\prime}, S_{2}^{\prime}, \ldots, S_{-b}^{\prime}$, we get a surface $S^{\prime}$ representing $\xi$. The genus of $S$ is $2 g-1$, so the genus of $S^{\prime}$ is

$$
-b(2 g-1)-(-b-1)=2 b g+2 b+1=a g+\frac{(a-1)(a+2 b-1)}{2} .
$$

Now let us assume that $a+2 b>0$. Move $S_{j}, 0 \leq j \leq a+2 b-1$, slightly in Interior $(D) \times \mathbb{C}$. We may assume that

- for $0 \leq i<j \leq a+2 b-1, S_{i}$ and $S_{j}$ intersect at only one point $p_{i, j}$ transversely, and $p_{i_{1}, j_{1}} \neq p_{i_{2}, j_{2}}$ if $\left(i_{1}, j_{1}\right) \neq\left(i_{2}, j_{2}\right)$.

- $S_{i}^{\prime}, 1 \leq i \leq-b$, intersects with $S_{j}, 0 \leq j \leq a+2 b-1$, at only one point $p_{i, j}^{\prime}$ transversely, and $p_{i_{1}, j_{1}}^{\prime} \neq p_{i_{2}, j_{2}}^{\prime}$ if $\left(i_{1}, j_{1}\right) \neq\left(i_{2}, j_{2}\right)$.

Consider this configuration of surfaces with $-b$ disjoint surfaces $S_{i}^{\prime}$ of genus $2 g-1$, and $(a+2 b)$ sections $S_{j}$ with only positive intersection points $p_{i, j}$ and $p_{i, j}^{\prime}$, the total number being

$$
(-b)(a+2 b)+\frac{(a+2 b)(a+2 b-1)}{2} .
$$

Do surgery on $(a+2 b)+(-b)-1=a+b-1$ of those to make the configuration into an irreducible nodal surface of genus $(-b)(2 g-1)+(a+2 b) g$ and representing $\xi$. Any surgery on the remaining one increases the genus by 1 . At the end we obtain an embedded surface representing $\xi$ and has genus exactly

$$
\begin{aligned}
& (-b)(2 g-1)+(a+2 b) g+(-b)(a+2 b)+\frac{(a+2 b)(a+2 b-1)}{2}-(a+b-1) \\
= & a g+\frac{(a-1)(a+2 b-2)}{2} .
\end{aligned}
$$

Remark 3.2. The formula (3.2) still holds in the case where $\Sigma$ has genus 0 ([7, [5]).

3.2. Blow ups. To further illustrate the power of the circle-sum construction, let us consider the minimal genus problem for blow ups of $N$.

We will employ the same strategy, but to keep the arithmetic simple we first make two general observations. 
Lemma 3.3. Let $\bigcup_{i=1}^{l} C_{i}$ be a connected configuration of embedded surfaces in an almost complex 4-manifold. Suppose each irreducible component satisfies the adjunction formula, and each pair intersects transversally and positively. Then the connected embedded surface $C$ obtained by surgery, which represents the class $\sum_{i=1}^{l}\left[C_{i}\right]$, also satisfies the adjunction formula.

Proof. We have by our assumption that

$$
\begin{gathered}
2 g\left(C_{i}\right)-2=K\left(\left[C_{i}\right]\right)+C_{i} \cdot C, \\
C \cdot C=\sum_{i=1}^{l} C_{i} \cdot C_{j}=\sum_{i=1}^{l} C_{i}^{2}+2 \sum_{1 \leq i<j \leq l} C_{i} \cdot C_{j} .
\end{gathered}
$$

On the other hand, by the positivity of the intersection, the genus of $C$ is

$$
\sum_{i=1}^{l} g\left(C_{i}\right)+\sum_{1 \leq i<j \leq l} C_{i} \cdot C_{j}-(l-1) .
$$

Therefore we have

$$
\begin{aligned}
2 g(C)-2 & =2\left[\sum_{i=1}^{l} g\left(C_{i}\right)+\sum_{1 \leq i<j \leq l} C_{i} \cdot C_{j}-(l-1)\right]-2, \\
& =\sum_{i=1}^{l}\left[2 g\left(C_{i}\right)-2\right]+2 \sum_{1 \leq i<j \leq l} C_{i} \cdot C_{j} \\
& =\sum_{i=1}^{l}\left[K\left(\left[C_{i}\right]\right)+C_{i} \cdot C_{i}\right]+2 \sum_{1 \leq i<j \leq l} C_{i} \cdot C_{j} \\
& =K([C])+C \cdot C .
\end{aligned}
$$

Lemma 3.4. Let $\coprod_{i=1}^{l} C_{i}$ be a configuration of disjoint embedded surfaces in an almost complex 4-manifold. Suppose each irreducible component satisfies the adjunction formula, and the circle-sum construction can be applied successively to get connected surfaces. Then the connected embedded surface $C$ obtained this way and representing the class $\sum_{i=1}^{l}\left[C_{i}\right]$ also satisfies the adjunction formula.

Proof. We have by our assumption that

$$
2 g\left(C_{i}\right)-2=K\left(\left[C_{i}\right]\right)+C_{i} \cdot C_{i}, \quad C \cdot C=\sum_{i=1}^{l} C_{i} \cdot C_{j} .
$$

On the other hand, by Theorem 2.1 the genus of $C$ is

$$
\sum_{i=1}^{l} g\left(C_{i}\right)-(l-1)
$$


Therefore we have

$$
\begin{aligned}
2 g(C)-2 & =2\left[\sum_{i=1}^{l} g\left(C_{i}\right)-(l-1)\right]-2 \\
& =\sum_{i=1}^{l}\left[2 g\left(C_{i}\right)-2\right] \\
& =\sum_{i=1}^{l}\left[K\left(\left[C_{i}\right]\right)+C_{i} \cdot C_{i}\right] \\
& =K([C])+C \cdot C .
\end{aligned}
$$

Definition 3.5. A good configuration in an almost complex 4-manifold is one as in Lemma 3.3 or in Lemma 3.4 .

Let $E_{i} \in H_{2}\left(N \# n \overline{\mathbb{C} P^{2}} ; \mathbb{Z}\right), 1 \leq i \leq n$, be represented by a line in the $i$ th $\mathbb{C} P^{2}$. Then $\left\{x, y, E_{1}, \ldots, E_{n}\right\}$ is a basis of $H_{2}\left(N \# n \overline{\mathbb{C} P^{2}} ; \mathbb{Z}\right)$ with

$$
E_{i} \cdot E_{i}=-1,1 \leq i \leq n, \quad x \cdot E_{i}=y \cdot E_{i}=0 .
$$

Hence a class $\xi=a x+b y-\sum_{i=1}^{n} c_{i} E_{i}$ has square

$$
a(a+2 b)-\sum_{i=1}^{n} c_{i}^{2} \geq 0 .
$$

To study the minimal genus problem for blow ups of $N$, it is convenient to use the notion of a reduced class.

Definition 3.6. A class $\xi=a x+b y-\sum_{i=1}^{n} c_{i} E_{i}$ in $N \# n \overline{\mathbb{C} P^{2}}$ is reduced if

$$
a \geq c_{1} \geq c_{2} \geq \cdots \geq c_{n} \geq 0
$$

In $[3$ it is shown that every class of nonnegative square can be transformed to a reduced class by orientation-preserving diffeomorphisms. Hence, to determine the minimal genera of classes of nonnegative square, it suffices to do so for reduced classes.

Remark 3.7. In fact we have used this idea in the proof of Theorem 3.1 (making $a \geq 0$ ). It is also shown in 2. that every class of nonnegative square has a unique reduced representative.

For a reduced class $\xi=a x+b y-\sum_{i=1}^{n} c_{i} E_{i}$, its minimal genus is bounded by the adjunction formula in [6],

$$
a g+\frac{(a-1)(a+2 b-2)}{2}-\sum_{i=1}^{n} \frac{c_{i}\left(c_{i}-1\right)}{2}
$$

where we use the standard canonical class whose Poincaré dual is

$$
P D(K)=-2 x+(2 g-1) y+\sum_{i} E_{i} .
$$

Remark 3.8. In fact the right hand side of (3.5) is the symplectic genus of the reduced class $\xi$ defined in $[3]$.

Theorem 3.9. Let $\xi=a x+b y-\sum_{i=1}^{n} c_{i} E_{i}$ be a reduced class of $N$ with $\xi^{2} \geq 0$ and $b<0$. Let $m \leq n$ be such that $c_{m}>0, c_{m+1}=\cdots=c_{n}=0$. Then the minimal genus of $\xi$ is given by (3.5) if 
(1) either $a+2 b \geq \sum_{i=1}^{m} c_{i}-m$,

(2) or $a+2 b=1$, and there is an $m^{\prime} \leq-b$ such that $c_{i}=2$ for $1 \leq i \leq m^{\prime}$ and $c_{i}=1$ for $m^{\prime}<i \leq m$.

Proof. Notice that the right hand side of (3.5) is given by the adjunction formula for a standard complex structure on $N \# n \overline{\mathbb{C} P^{2}}$ with $K$ given by (3.6). So by Lemmas 3.3 and 3.4 it suffices to show that $\xi$ is represented by a good configuration with respect to (3.6).

Let

$$
S_{j}, \quad 0 \leq j \leq a+2-1, \quad \text { and } \quad S_{i}^{\prime}, \quad 0 \leq i \leq-b,
$$

be as in Theorem 3.1 .

Let us first deal with case (1). If $a+2 b=0$, we then must have $c_{1}=c_{2}=\cdots=$ $c_{m}=1$. We have seen that $a x+b y$ is represented by a connected embedded surface $S^{\prime}$ in $N$ with genus $a g-(a-1)$. Choose $m$ distinct points $p_{1}, \ldots, p_{m}$ on $S^{\prime}$ and $n-m$ distinct points $p_{m+1}, \ldots, p_{n}$ on $N-S^{\prime}$ and blow up at $p_{1}, \ldots, p_{n}$. Then the proper transform of $S^{\prime}$ in $N \# n \overline{\mathbb{C} P}^{2}$ is an embedded surface of required genus.

From now on let us assume that $a+2 b>0$. Let $c_{m^{\prime}}>1$ and $c_{m^{\prime}+1}=\cdots=c_{m}=$ 1. Move $S_{j}, 0 \leq j \leq \sum_{i=1}^{m} c_{i}-m$, slightly in Interior $(D) \times \mathbb{C}$ such that $c_{i}-1$ of them pass through a point $p_{i}$ in $S_{1}^{\prime}$ transversally to each other for $0 \leq i \leq m^{\prime}$, and $p_{1}, \ldots, p_{m^{\prime}}$ are distinct. Choose distinct points $p_{m^{\prime}+1}, \ldots, p_{m}$ on $S_{1}^{\prime}-\left\{p_{1}, \ldots, p_{m^{\prime}}\right\}$, and distinct points $p_{m+1}, \ldots, p_{m}$ in $N$ outside $S_{j}$ and $S_{i}^{\prime}$. Then by blow up at $p_{1}, \ldots, p_{n}$, and circle-sum $S_{1}^{\prime}, \ldots, S_{-b}^{\prime}$, we get a configuration of embedded surfaces in $N \# n \overline{\mathbb{C} P^{2}}$ representing $\xi$. Notice that each irreducible component satisfies the adjunction formula.

If $-b>1$, since each $S_{j}$ intersects with $S_{-b}^{\prime}$ at one point, the configuration is connected, and hence good in the sense of Lemma 3.3. It is also the case if $-b=1$ and $a+2 b>\sum_{i=1}^{m} c_{i}-m$.

If $-b=1$ and $a+2 b=\sum_{i=1}^{m} c_{i}-m$, the configuration is not connected. When $m^{\prime}=1$, it has $a+2 b+1$ components which are the proper transforms of $S_{0}, \ldots, S_{a+2 b-1}$ and $S_{1}^{\prime}$. If $m^{\prime}>1$, it has two components, one from $S_{j}, 0 \leq j \leq$ $a+2 b-1$, another being $S_{1}^{\prime}$. Certainly both configurations are good in the sense of Lemma 3.4.

Finally let us deal with case (2). Since $a+2 b=1, S_{0}$ and the circle-sum of $S_{1}^{\prime}, \ldots, S_{-b}^{\prime}$ have $-b$ positive intersection points. As $m^{\prime}$ is assumed to be less than $-b$, we can choose $m^{\prime}$ of them and label them as $p_{1}, \ldots, p_{m^{\prime}}$. Fix distinct points $p_{m^{\prime}+1}, \ldots, p_{m}$ on $S_{0}$ away from the intersection points. Also fix distinct points $p_{m+1}, \ldots, p_{n}$ outside $S_{0}$ and $S_{i}^{\prime}, 0 \leq i \leq-b$. Blowing up at $p_{i}, 0 \leq i \leq n$, to obtain a configuration of embedded surfaces in $N \# n \overline{\mathbb{C} P^{2}}$, each irreducible component satisfying the adjunction formula. The configuration represents $\xi$ from our assumption on the $c_{i}$. Moreover, the configuration is good, because it is connected if $-b>m^{\prime}$ and is circle-summable if $-b=m^{\prime}$.

Let us illustrate the power and the limitation of Theorem 3.9 in the case $n=1$. Then the assumption (1) is $a+2 b \geq c-1$. Notice that in this case the assumption (2), which is $a+2 b=1, c=2$, actually implies the assumption (1) and thus can be ignored. One can easily check that, if $a \leq 7$, then any reduced class with a nonnegative square satisfies $a+2 b \geq c-1$. 
Example 3.10. The simplest reduced class in $N \# \overline{\mathbb{C} P^{2}}$ not satisfying the condition $a+2 b \geq c-1$ is $\xi=8 x-3 y-4 E$. Notice that $\xi \cdot \xi=0$, and its symplectic genus is $8 g-6$ (cf. Remark 3.8). We do not know whether $8 g-6$ is the minimal genus of $\xi$.

Example 3.11. The reduced class $\xi=9 x-3 y-5 E$ has $\xi^{2}=2$ and $a+2 b=3<$ $c-1=4$. According to Theorem B in $[6$, if the symplectic genus of a reduced class $\alpha$ is no more than $\alpha^{2}+1$, then the symplectic genus is the minimal genus of $\alpha$. The symplectic genus of $\xi$ is $9 g-6$. In particular, if $g=1$, then $\xi^{2}+1=2+1$ is no less than the symplectic genus $9-6=3$. Therefore the minima genus of $\xi$ is 3 in this case. It would be interesting to find a direct construction using circle-sum.

Remark 3.12. It is expected that (3.5) also often gives the minimal genus when $b \geq$ 0 . We concentrate on the case $b<0$ in Theorem 3.9 as the circle-sum construction is essential in this case. In the case $b \geq 0$ we can sometimes determine $g_{\xi}$ without using the circle-sum construction. For example, if $n=1$ and $b \geq 0$, then the configuration of $b$ fibers, $c$ proper transforms of the zero sections, and $(a-c)$ zero sections is already a good configuration. For more general $n$ in the case $b \geq 0$, see [2].

\section{REFERENCES}

1. R. Friedman \& J. Morgan, Algebraic surfaces and Seiberg-Witten invariants, J. Algebraic Geom. 6(1997), 445-479. MR.1487223 (99b:32045)

2. H. Gao \& X. Zhao, Minimal genus problem in ruled manifolds, preprint.

3. B. H. Li \& T. J. Li, Symplectic genus, minimal genus and diffeomorphisms, Asian J. Math. 6(2002), 123-144. MR1902650 (2003c:57027)

4. B. H. Li \& T. J. Li, Minimal genus embeddings of surfaces in $S^{2}$-bundles over surfaces, Math. Res. Lett. 4(1997), 379-394. MR.1453068 (98h:57062)

5. B. H. Li \& T. J. Li, Minimal genus smooth embeddings in $S^{2} \times S^{2}$ and $C P^{2} \# n \overline{C P}^{2}$ with $n \leq 8$, Topology 37(1998), 573-594.

6. T. J. Li \& A. K. Liu, Symplectic structure on ruled surfaces and generalized adjunction formula, Math. Res. Lett. 2(1995), 453-471. MR1355707 (96m:57052)

7. D. Ruberman, The minimal genus of an embedded surface of nonnegative square in a rational surface, Proc. of Gokova Geometry-Topology conference, 1995, 129-134.

Key Laboratory of Mathematical Mechanization, Academy of Mathematics and Systems Science, Chinese Academy of Sciences, Beijing 100080, China

E-mail address: Libh@amss.ac.cn

School of Mathematics, University of Minnesota, Minneapolis, Minnesota 55455

E-mail address: tjli@math.umn.edu 